\title{
Enterotoxigenic Escherichia coli detection using the design of a biosensor
}

\author{
V. Lakshman Narayana* and A. Peda Gopi \\ Vignan's Nirula Institute of Technology \& Science for Women, Peda Palakaluru, Guntur 522009, Andhra Pradesh
}

Corresponding Author Email: lakshmanv58@gmail.com

\begin{abstract}
The food industry and clinical analysis, among other sectors, require the development of techniques and devices that detect pathogens, while the development of implantable devices needs biocompatible materials with low degradation in biological environment to increase the lifetime of the device. Throughout this work, hydrogenated amorphous silicon-carbon alloy is proposed, obtained, characterized and incorporated into the development of a proposed interdigitated microelectrode array (PIMA) to capture the bacteria of enterotoxigenic Escherichia coli (E. coli, ETEC). a-SixC1-x:H is obtained by the technique of plasma-enhanced chemical vapor deposition (PECVD) using methane and silane as precursor gases under high hydrogen dilution and low power density in order to improve its biocompatibility. Functionally the PIMA is a transducer based on electrical impedance, namely the capture of E. coli bacteria causes changes in the electrical properties of the medium between and on the microelectrodes of the array, which are associated with changes in electrical impedance. The simulations were made with the purpose of knowing the operation that the PIMA would have under operating conditions (with bacterial environment) and of analyzing the design aspects that could affect or increase the sensitivity of the array.
\end{abstract}

Keywords: food Industry, biological environment, enterotoxigenic, microelectrode array, vapor deposition Received: June-17-2020, Accepted: July-26-2020, https://doi.org/10.14447/jnmes.v23i3.a02

\section{INTRODUCTION}

A biosensor is a device that integrates the biochemical recognition of biological compounds (proteins, DNA, viruses, bacteria, cells, etc.) with a signal transducer. Therefore, the specification of a biosensor comprises the recognition method and the transduction method. When any of these elements is changed, the biosensor changes, then there are as many biosensors as there are possible combinations of recognition and transduction methods. Among the most common recognition methods are ion recognition, affinity interaction, nucleic acid hybridization, enzymatic action and recognition using cells and tissues of biological origin. The list of transducers, instead, is a bit longer and starts by differentiating between free-label and label-based transducers. In biosensors in which any compound in the recognition process is not detectable, other compound can be used to label one or most product compounds and to do them detectable by the corresponding transducer. In this case the transducer is known as label-based.

The development of materials for biosensors involves a sequence of steps, which comprise from the selection of the method of obtaining of the material to the tests on device. These steps must be always oriented according to the required properties of the material. Depending on the application, morphological, structural, chemical, optical, mechanical, electrical, among other properties should be adjusted. The excellent mechanical, chemical, electrical and optical properties of hydrogenated amorphous silicon-carbon alloy (aSixC1-x:H) makes this material an excellent candidate for the fabrication of devices whose structure requires mechanical stability, high electrical resistivity, transparency to sunlight, thermal stability and resistance to corrosion and wear.

The deposition of a-SixC1-x:H by PECVD is carry out in a reactor with the necessary gases in the chamber of deposition. The reactor can be of two types, capacitively or inductively coupled. On the one hand, a reactor coupled capacitively consists of a pair of flat parallel plates, with one plate operating as support for the substrate on which the film will be deposited. On the other hand, a reactor coupled inductively is surrounded by wire, which is part of the winding inductor, which gives rise to the electromagnetic field inside the deposition chamber when it is excited by the supply.

The pressure in the chamber during deposition influences the density of reactive species and hence the deposition rate; under low pressure, although the reactive species are more energetic owing to its longer mean free path, the density of species in the plasma is lower, which reduces the deposition rate. Moreover, it has been reported that at low pressures the films have low uniformity [6]. The substrate temperature, instead, affects directly the morphology of the film. Increasing the substrate temperature, the kinetic energy for migration of absorbed molecules increases, and thus the reactive species can find the site energetically more favorable during deposition, improving the structure of the film (lower degree of disorder) and reducing the residual stress.

At this point it is noteworthy that as the substrate temperature increases, the hydrogen promotes the conversion of free radicals in molecules [7], and simultaneously varies its incidence due to desorption from the surface [8]; therefore, not necessarily an increase in temperature will increase the deposition rate and the conductivity of the film. The composition of the film is controlled by the ratio of precursor 
gases (defined as the ratio between the flow of methane or acetylene, and the total flow of precursor gases). The optimization of the film composition is also a surface related

\section{APPLICATIONS OF A-SIXC1-X:H IN BIOSENSORS}

In the last 20 years, looking for new materials that improve the performance of biosensors and allow implantation into living organisms for long periods of time, a-SixC1-x:H has emerged as one of the candidates. In terms of compatibility with current manufacturing technology based on silicon, aSixC1-x:H is deposited at low temperatures, which do not affect the previous stages of the manufacturing process. As for the function within the structure of a biosensor, the properties of a-SixC1-x:H can be adjusted with the deposition parameters, giving versatility to the design. In addition, aSixC1-x:H is chemically stable and its surface can be bio functionalized.

\section{MATERIALS AND METHODS}

The design of the proposed inter digitated microelectrode array (PIMA) is made according to the distribution of the electric field, the results of previous published works and taking into account that the sensing area is enough to house around 5x106 colony-forming units (CFU). In order to know the electrical behavior under the design parameters, simulations of the PIMA through the platform of the CoventorWare ${ }^{\circledR}$ software will be carried out. At the end of this chapter and in accordance with the results of the simulations, the more suitable properties of a-SixC1-x:H films for this and other biological applications will be specified.

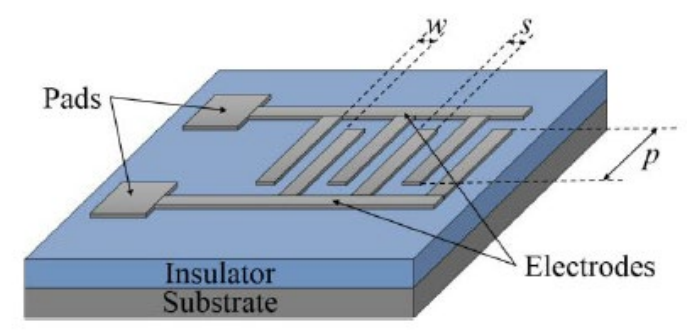

Figure 1. Layout of an interdigitated microelectrode array

Based on this, this work proposes an IMA that uses the whole surface over the array (the area between and on top of metal microelectrodes). To achieve it a layer acting as support for the bio-layer would be placed on the metal microelectrodes. But this is not enough, the surface of this layer would also have to be compatible with the biofunctionalization process. Thinking about this, this work proposes hydrogenated amorphous silicon-carbon alloy as material to be biofunctionalized.

In the proposed work the bacterium of Escherichia coli (E. coli) using the PIMA is detected. E. coli is a prokaryotic organism, facultative anaerobic, and moves through flagella which surround its body. It is one of the most studied bacteria and its ubiquitous nature allows to find it everywhere, especially in the intestines of humans and other animals. Humans become infected via gastrointestinal by eating contaminated and poorly cooked food and via urinary by bad composition aspect. Accordingly, appropriate new materials development and utilization are required.

habits. When a human is infected by E. coli, it can cause him severe intestinal and extra-intestinal infections. Six pathogenic strains of E. coli are known: enterotoxigenic E. coli (ETEC), enteroinvasive E. coli (EIEC), enteropathogenic E. coli (EPEC), enterohemorrhagic E. coli (EHEC), enteroaggregative E. coli (EAEC) and diffuse-adhering E. coli (DAEC). Specifically, the strain to be detected in this work is the ETEC.

The fabrication process of the PIMA requires two masks, one mask to define the array and other one to protect the array and to etch the B-doped a-SixC1-x:H on pads of the array. The first one will be called "Level 1 mask", and the second one will be called "Level 2 mask". The Level 1 mask includes not only the design of the PIMA, but also some variations in design parameters, position of the pads and $\mathrm{Ti}$ shielding. Arrays with finger width and spacing of 5, 10 and $15 \mu \mathrm{m}$, with symmetric and asymmetric pads and without and with shielding, are in the design of the Level 1 mask. The shielding is the same layer surrounding the array and the pads and distanced from them $50 \mu \mathrm{m}$. Therefore, the Level 1 mask includes twelfth different arrays in total. The Level 2 mask is simpler and includes only squares of $3.2 \mathrm{~mm}$ side, which correspond to the areas of the arrays in Level 1 mask (the squares do not include pads). In addition, both masks include structures of characterization and alignment marks. The crossbridge structure, like its name suggest, is formed by two cross or van der Paw structures and one bridge structure.

With the purpose of improving the sensitivity of these arrays, the deposition of a thin layer of a-SixC1-x:H $(50 \mathrm{~nm})$ on microelectrodes was proposed to increase the sensing area. However, this layer involves changes in the operation and therefore in the model of the array, increasing its complexity. One disadvantage of this proposal is that both the a-SixC1-x:H layer and the bio-layer are dielectrics which do not reach the value of the permittivity of the double dielectric layer and thus decrease the range of the plateau of the impedance spectrum.

To overcome this effect, it is suggested that these layers have a high permittivity and should be as thin as possible. Another option is to reduce the resistance associated with each layer, making it much lower than the respective reactance in the frequency range of operation (for example the plateau of the impedance spectrum), which would allow to neglect the reactance. However, the resistances associated would be added to the serial connection of the resistance of the titanium electrodes and the solution, reducing the sensitivity of the array. Despite that, it is expected that the gain in sensitivity when the whole sensing area of the array, not only the area between microelectrodes, is used to entrap bacteria, it overcomes the loss of sensitivity by resistances in serial connection. For this reason, the first simulations were designed to study the effect of the conductivity of the a-SixC1$\mathrm{x}$ :H layer on top of microelectrodes, using reported values.

Parameters such as thickness and surface roughness are used to evaluate the morphology of the films and to characterize the deposition processes. Measurement of the thickness can be done using the techniques of profilometry or ellipsometry. Profilometry, either optical or contact allows to scan the surface and thus to obtain the surface topology. First the sample must have a step on the surface whose depth corresponds to the thickness of the film. Contact profilometry makes contact with the sample using a tip. As the tip moves 
through the sample, the head holding the tip moves vertically keeping the force that the sample exerts on the tip; thus, by passing through the step, the depth of the fall of the head is measured, which corresponds to the thickness of the film [78]. Otherwise, ellipsometry is an optical nondestructive technique that measures indirectly the thickness and the refractive index. An example of data setting procedure is shown in Figure 2.

The series attachment of impedance elements to the cell membrane and cytoplasm can be used to electrically reflect the bacteria. The cell membrane is characterised by the parallel between its ion flow control mechanisms and its structurerelated capacitance (CM) associated with a resistance (RM). The cytoplasm, which is about $80 \%$ of the water, can also be expressed by its resistivity and permittivity associated with a parallel relation of resistor (RC) and capacitance (CC). This bacterium electric model allows the electric PIMA model to be established in solution with bacterial interactions.

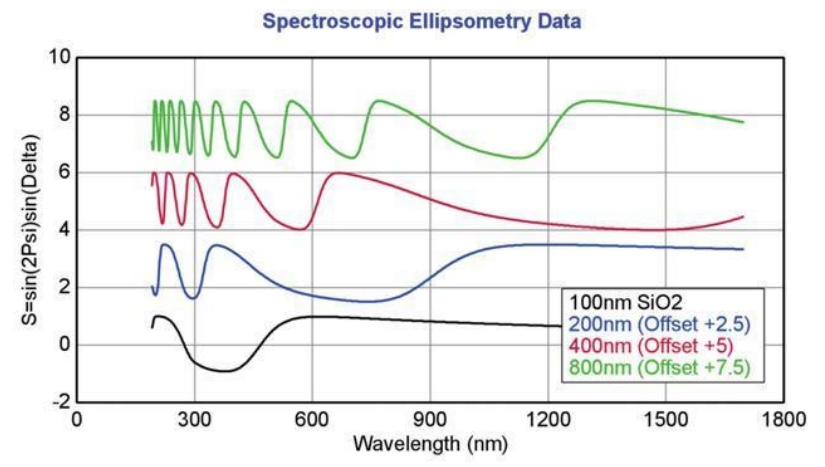

Figure 2. Spectroscopic Ellipsometry Data representation

Among biosensors, the immunosensor is defined as a class of sensors that use biological affinity interactions in which the affinity reagent functions as a recognition receptor and another, the target compound, operates as an analyte. This recognition approach is based on the recognition process used by the immune system of living organisms to neutralise foreign objects. Under this assumption, the main compounds are antibodies, generally referred to as Ab. An antibody is a long protein used by the immune system to detect and neutralise foreign particles and microorganisms, such as bacteria and viruses. The most common proteins are immunoglobulins (Ig) found in blood plasma. The antibody recognises only a portion of the foreign object that is considered an antigen and denoted by Ag. When the antigen is identified by the immune system, it releases antibodies that bind several non-covalent bonds to the antigen, forming an immunochemical complex denoted by $\mathrm{Ab}$ : Ag. Before continuing with the binding process, an outline of the general structure of the antibody is helpful. The first is a fixed domain, and in this case the sequence of amino acids is the same for antibodies of the same class as immunoglobulins $\mathrm{G}$ ( $\mathrm{IgG})$, while in the second, a variable domain, the sequence of amino acids is modified to identify a particular antigen.

\section{CONCLUSION}

Depending on the application, morphological, structural, chemical, optical, mechanical, electrical, among other properties should be adjusted. The excellent mechanical, chemical, electrical and optical properties of hydrogenated amorphous silicon-carbon alloy (a-SixC1-x:H) makes this material an excellent candidate for the fabrication of devices whose structure requires mechanical stability, high electrical resistivity, transparency to sunlight, thermal stability and resistance to corrosion and wear. The impedance spectra obtained in the range from $10 \mathrm{kHz}$ to $100 \mathrm{MHz}$ show that if the a-SixC1-x:H layer has a lower conductivity than 10-3 $\mathrm{S} / \mathrm{cm}$, then the sensitivity of the array is significantly reduced. The design of the proposed interdigitated microelectrode array (PIMA) is made according to the distribution of the electric field, the results of previous published works and taking into account that the sensing area is enough to house around 5x106 colony-forming units (CFU).

\section{REFERENCES}

[1] Ibrahim, M., Claudel, J., Kourtiche, D., Nadi, M. (2013). Geometric parameters optimization of planar interdigitated electrodes for bioimpedance spectroscopy. Journal of Electrical Bioimpedance, 4: 13. https://doi.org/10.5617/jeb.304

[2] Radke, S.M., Alocilja, E.C. (2004). Design and fabrication of a microimpedance biosensor for bacterial detection. IEEE Sensors Journal, 4(4): 434-440. https://doi.org/10.1109/JSEN.2004.830300

[3] Radke, S.M., Alocilja, E.C. (2005). A high density microelectrode array biosensor for detection of E. coli O157:H7. Biosensors and Bioelectronics, 20(8): 16621667. https://doi.org/10.1016/j.bios.2004.07.021

[4] Coletti, C. (2007). Silicon Carbide Biocompatibility, Surface Control and Electronic Cellular Interaction for Biosensing Applications. Ph. D. Thesis. University of South Florida.

[5] Rajeshwar, K. (2002). Fundamentals of semiconductor electrochemistry and photoelectrochemistry. Encyclopedia of Electrochemistry, 6: 1-51. https://doi.org/10.1002/9783527610426.bard060001

[6] Tiginyanu, I., Langa, S., Foell, H., Ursachi, V. (2009). Porous III-V semiconductors. On-line book. Section 2.5.2. Available from: http://www.porous-35.com/

[7] Olthuis, W., Streekstra, W., Bergveld, P. (1995). Theoretical and experimental determination of cell constant of planar-interdigitated electrolyte conductivity sensors. Sensor and Actuator B: Chemical, 24(1-3): 252256. https://doi.org/10.1016/0925-4005(95)85053-8

[8] Rodríguez-Angeles, G. (2002). Diagnosis and main characteristics of Escherichia coli pathogenic groups. Salud pública Méx, 44(5): 464-475.

[9] Asami, K., Hanai, T., Koizumi, N. (1980). Dielectric analysis of Escherichia coli suspensions in the light of the theory of interfacial polarization. Biophys Journal, 31(2): 215-228.

[10] Lvovich, V.F. (2012). Impedance Spectroscopy Applications to Electrochemical and Dielectric Phenomena. 1st edition, Wiley, Hoboken.

[11] Bakthavathsalam P, Rajendran VK, Mohammed JAB (2012). A direct detection of Escherichia coli genomic DNA using gold nanoprobes. J Nanobiotechnol 2012;10:1-10.

[12] Fauchet, P. M. (2009). Porous Silicon Optical LabelFree Biosensors. In Device Applications of Silicon Nanocrystals and Nanostructures, 293-323. https://doi.org/10.1007/978-0-387-78689-6_1. 